\title{
Error Corrected References and Acceleration of Norm Optimal Iterative Learning Control
}

\author{
David $\mathrm{H}$ Owens ${ }^{1}$ and Bing $\mathrm{Chu}^{2}$
}

\begin{abstract}
Iterative learning control is a control design method for high performance tracking applications. In this paper a simple mechanism for accelerating the convergence of a well-known Norm Optimal Iterative Learning Control (NOILC) Algorithm is presented by modifying the reference signal each iteration using the previously measured tracking error. The change is equivalent to successive application of a gradient and NOILC iteration. The change is interpreted in terms of the spectrum of the error update operator and the annihilation of spectral components of the error signal. Convergence of the proposed algorithm is analysed rigorously and numerical examples are given to demonstrate the effectiveness of the proposed method.
\end{abstract}

\section{INTRODUCTION}

Iterative Learning Control (ILC) is now well established as part of the control scene and many papers have been published [1], [2] plus several texts [3]-[5]. The area of study poses all of the challenges of Control Theory with the added complication that the system to be controlled is required to track a given reference (or demand) signal on a finite time interval and the physical controlled process operates in a repetitive manner. Typical applications examples include robotic systems [6], chemical batch processing [7], [8] and stroke rehabilitation [9]. The repetition (or iteration as it is typically called) is both a complication and an opportunity. It complicates the control design but also offers the opportunity to use measured input/output (and other) data from previous iterations to improve performance. Performance improvement is typically expressed in terms of reducing the tracking error and has the ultimate objective of producing higher accuracy than can be achieved by feedback control alone. In a mathematical sense, the iterations/repetitions are allowed to be infinite in number. In practice, iterations will be finite in number and the control improvement process will be terminated when the tracking accuracy has been reduced to desired levels.

A number of design paradigms [10]-[25] have been suggested and considerable insight has been obtained into the nature of the control problem and performance limitations. Issues of performance have focussed to a large degree on the convergence of the iterative process. Clearly convergence to a desired solution is part of the requirement but, as each iteration is a physical process in the real world (often

\footnotetext{
${ }^{1}$ David H Owens is with Department of Automation, Zhengzhou University, Zhengzhou, 450001, P. R. China and Department of Automatic Control and Systems Engineering, The University of Sheffield, Mappin Street, Sheffield S1 3JD d.h. owen@ shef .ac.uk

${ }^{2}$ Bing Chu is with school of Electronic and Computer Science, University of Southampton, Southampton, SO17 1BJ, UK b. chu@ soton. ac. uk
}

involving the organization of machines and operators), the speed of this convergence is also important if time and cost are to be within acceptable bounds. This paper focusses on the use of the Norm Optimal Iterative Learning Control (NOILC) algorithm proposed by Amann et al [16] and brought together in the recent book by Owens [26] . This class of algorithms constructs control inputs for each iteration by minimizing a quadratic performance index. Further details are provided in later sections but the essential properties of NOILC for linear processes are that control improvements are guaranteed theoretically from iteration to iteration but that the rate of convergence depends upon the choice of parameters in the index. For state space systems, the general result is that fast convergence requires the use of high gain state feedback. High gain may not be appropriate for many applications and other mechanisms would be preferable.

In this paper, improved convergence rates are shown to be possible without high gain control by retaining the NOILC methodology but adding the simple mechanism of changing the reference signal $r$ from iteration to iteration in a systematic manner. In mathematical terms, the reference $r$ is replaced in NOILC calculations by a sequence of references $\left\{r_{k}\right\}_{k \geq 0}$ where $r_{k}$ denotes the reference signal used on iteration $k$. The NOILC parameters can then be chosen to avoid excessively high gains in an implementation and the reference signals chosen to improve convergence rates. The changes in the reference signals chosen are related to the tracking error observed on the previous iteration in a simple manner. The development in the paper initially summarizes the basic ideas of NOILC using the operator formulation used by Owens [26]. This has the advantage of notational simplicity and generality as many types of linear dynamics can be analysed and controlled. This is followed by a discussion of the related Gradient ILC algorithm in the form given by Owens [26]. It is noted that,

1) if the NOILC algorithm and the Gradient algorithm are applied on alternate iterations, the basic convergence properties of NOILC are still achieved

2) and that the two iterations can be combined to form a new, simplified algorithm that takes the form of NOILC with variable reference signals.

Not only is the algorithm more rapidly convergent than the use of NOILC alone, the choice of a free parameter in the new algorithm can be approached using the concept of spectral annihilation introduced by Owens in [26]. For state space systems, this offers the opportunity to focus improved convergence rates into selected frequency bands. 
The object used to provide the results is the operator relating the error on successive iterations. More precisely, it is the spectrum of this operator that reveals the link to convergence rates. This opens up the possibility of more complex updates for the reference signals constructed to achieve number of objectives. The details of this process are too complex and lengthy for this paper which concentrates on providing the basic algorithm and demonstrating its capabilities using numerical examples. More general results and a characterization of the robustness of the scheme will form the content of a future paper to be submitted to a journal.

\section{UNDERLYING OPTIMIZATION ALGORITHMS}

\section{A. Models, Signals and Operators}

The analysis aims to cover as many applications situations as possible. This is achieved by following the original methodology of Norm Optimal Iterative Learning Control (NOILC) proposed in Amann et al [16] and used more extensively in the text [26] by Owens. More precisely, the plant dynamics is assumed to be linear and described by a bounded linear operator $G$ mapping a real Hilbert space $\mathcal{U}$ of input signals $u \in \mathcal{U}$ into a real Hilbert space $\mathcal{Y}$ of output signals $y \in \mathcal{Y}$. The inner product in $\mathcal{U}$ (resp. $\mathcal{Y}$ ) is denoted by $\langle u, v\rangle_{\mathcal{U}}\left(\operatorname{resp} .\langle y, w\rangle_{\mathcal{Y}}\right)$. The induced norms in $\mathcal{U}$ (resp. $\left.\mathcal{Y}\right)$ are then given by $\|u\|_{\mathcal{U}}=\langle u, u\rangle_{\mathcal{U}}^{\frac{1}{2}}$ (resp. $\|y\|_{\mathcal{Y}}=\langle y, y\rangle_{\mathcal{Y}}^{\frac{1}{2}}$ ).

The model equations are represented in the form

$$
y=G u+d
$$

where $d \in \mathcal{Y}$ represents the initial condition or similar effects in the system dynamics. As noted in the text [26] by Owens, the operator description covers many situations of practical interest including linear continuous time state space models, sampled data and multi-rate sampling systems and problems where control signals have specific structure exemplified by a requirement that it is continuous and piecewise linear with discontinuities only allowed at specific time instants. The inner products and norms are design variables that can be chosen to reflect physical properties of the system, the control objective and/or the performance of the iterative algorithm to be used.

\section{B. Iterative Learning Control (ILC)}

Iterative Learning Control (ILC) considers a repetitive task whose ultimate objective is to ensure that the output from the system given by equation (1) tracks a given reference signal $r \in \mathcal{Y}$ arbitrarily accurately. Using the formal definition in the text by Owens [26], an ILC algorithm considers an infinite sequence of iterations labelled by the iteration index $k=0,1,2,3, \cdots \cdots$. The index with index $k=0$ is the zeroth iteration and is characterized by the choice of an initial control $u_{0}$, measurement of the corresponding output $y_{0}$ and computation of the error signal $e_{0}=r-y_{0}$. For future iterations, the algorithm uses measurement data from iterations $k^{\prime} \leq k$ plus, possible, model-based calculations, to construct an input $u_{k+1}$ for use on the next iteration $k+1$.
The result of this input is the output $y_{k+1}$ and tracking error $e_{k+1}=r-y_{k+1}$.

The design issue for ILC is the computational nature of the construction of $u_{k+1}$ and it is here that different approaches can be taken. For the purposes of this paper, the major requirement of the computation is that,

$$
\lim _{k \rightarrow \infty}\left\|e_{k}\right\| \mathcal{Y}=0
$$

which expresses the ideal outcome that the ultimate value of the tracking error is zero. Convergence of the input sequence $\left\{u_{k}\right\}_{k \geq 0}$ is also desirable but plays little role in this paper due to space limitations.

\section{Norm Optimal Iterative Learning Control (NOILC)}

Norm Optimal Iterative Learning Control (NOILC) [16], [26] now has a number of interpretations and extensions. In this paper we consider its basic and simplest form. More precisely, given data $\left(u_{k}, y_{k}\right)$ on the $k^{\text {th }}$ iteration, NOILC constructs the input $u_{k+1}$ to be used on iteration $k+1$ by minimizing the quadratic objective function

$$
J(u)=\|e\|_{\mathcal{Y}}^{2}+\epsilon^{2}\left\|u-u_{k}\right\|_{\mathcal{U}}^{2}
$$

subject to the constraints given by the system dynamics (1). If $G$ is a state space model and $\mathcal{Y}$ and $\mathcal{U}$ are $L_{2}[0, T]$ spaces, this problem is simply a linear quadratic optimal control problem with a familiar Riccati-style solution.

Properties of the algorithm include the important convergence properties listed below

1) the error sequence satisfies the update relationship $e_{k+1}=L e_{k}, k \geq 0$ where the operator $L$ is given by

$$
L=\left(I+\epsilon^{-2} G G^{*}\right)^{-1}
$$

and $G^{*}$ is the adjoint operator of $G$,

2) the error signal norm sequence is monotonic in the sense that

$$
\left\|e_{k+1}\right\| \mathcal{Y}<\left\|e_{k}\right\| \mathcal{Y}, \quad k \geq 0
$$

3 ) and the error signal converges to the signal given as the orthogonal projection of $e_{0}$ onto the subspace of $\mathcal{Y}$ defined as the kernel of $G G^{*}$.

The parameter $\epsilon^{2}$ is a measure of the relative weight given to the error and change in control signal. As $\epsilon^{2}>0$ reduces, the 'controller gain' increases and any feedback implementation is of a high gain form.

\section{Gradient-based Iteration}

NOILC can have a complex computational structure but a simplification is possible using gradient ideas. Using the approach of Owens in [26], a gradient algorithm uses the update formula,

$$
u_{k+1}=u_{k}+\beta_{k+1} G^{*} e_{k}, \quad k \geq 0 .
$$

Here $G^{*}$ is the adjoint operator of $G$ and $\beta_{k+1}>0$ is a scalar gain parameter (the step length in normal gradient terminology). The error update equation in this case is

$$
e_{k+1}=\left(I-\beta_{k+1} G G^{*}\right) e_{k}, \quad k \geq 0 .
$$


The algorithm retains the monotonicity property if, for all $k \geq 0$,

$$
0<\beta_{k+1}<2\left\|G^{*}\right\|^{-2}
$$

where $\left\|G^{*}\right\|$ is the norm of the operator $G^{*}: \mathcal{Y} \rightarrow \mathcal{U}$. The NOILC characterization of the limit also holds true in the case of iteration independent gains.

Note 1: If $G$ is a matrix, with singular values $0 \leq \cdots \leq$ $\sigma_{2}^{2} \leq \sigma_{1}^{2}$ then its norm is the largest singular value $\sigma_{1}^{2}$. If $G$ is a single-input, single-output (SISO) discrete state space model characterized by matrices $A, B, C$ and time series of length $N+1$ and $\mathcal{Y}$ and $\mathcal{U}$ are the Hilbert spaces $R^{N+1}$ with inner products

$$
\langle y, w\rangle_{\mathcal{Y}}=Q y^{T} w, \quad\langle u, v\rangle_{\mathcal{U}}=R u^{T} v
$$

where $Q$ and $R$ are strictly positive scalars, then an upper bound for the norm is obtained from the inequality [26]

$$
\|G\|^{2}=\left\|G^{*}\right\|^{2} \leq \sup _{|z|=1} R^{-1} Q|G(z)|^{2}
$$

where $G(z)$ is the transfer function of the system. More general, multi-input,multi-output (MIMO) expressions can be found in [26].

\section{ERROR CORRECTED REFERENCES AND ACCELERATION OF NOILC}

\section{A. An Error Corrected Reference Algorithm}

NOILC and Gradient methods are related and, in principle, the choice of algorithm can change from iteration to iteration. For example, if iteration one is a gradient iteration and iteration two is a NOILC iteration, the following error update equation and monotonicity property follows from (4) and (7)

$$
e_{2}=L\left(I-\beta_{1} G G^{*}\right) e_{0}, \quad\left\|e_{2}\right\|_{\mathcal{Y}}<\left\|e_{0}\right\|_{\mathcal{Y}}
$$

The corresponding input equations are

$$
u_{1}=u_{0}+\beta_{1} G^{*} e_{0}, \quad u_{2}=u_{1}+\epsilon^{-2} G^{*} e_{2} .
$$

The crucial observation is that the two input equations can be combined to give

$$
u_{2}=u_{0}+\epsilon^{-2} G^{*}\left(\epsilon^{2} \beta_{1} e_{0}+e_{2}\right) .
$$

This demonstrates that

Theorem 1: A gradient iteration followed by a NOILC iteration can be combined as one NOILC iteration with the reference signal $r$ replaced by $r+\epsilon^{2} \beta_{1} e_{0}$.

The same argument can be applied to future pairs of iterations and proves the following result. Note the change in indexing used to reflect the combined gradient and NOILC iterations.

An Error Corrected Reference Algorithm: Let $r$ be the reference signal that is to be tracked. In addition, choose $\left\{\beta_{k+1}\right\}_{k \geq 0}$ to be an infinite sequence of scalar gains in the range defined by (8) and define the sequence of error corrected reference signals

$$
r_{k+1}=r+\epsilon^{2} \beta_{k+1} e_{k}, \quad k \geq 0
$$

with

$$
e_{k}=r-y_{k}, \quad k \geq 0
$$

Consider the error corrected reference NOILC algorithm defined by the iterations and computations described below starting with index $k=0$ :

Step 1 Given the data $u_{k}, y_{k}, e_{k}$, compute $r_{k+1}$ and construct the form of the input $u_{k+1}$ by minimizing the performance index

$$
J_{k+1}(u)=\left\|r_{k+1}-y\right\|_{\mathcal{Y}}^{2}+\epsilon^{2}\left\|u-u_{k}\right\|_{\mathcal{U}}^{2}
$$

subject to the constraint of the plant dynamics (1). Note that $J_{k+1}$ is a modified form of the original NOILC performance index (3) with $r$ replaced by $r_{k+1}$.

Step 2 Use this information to obtain the plant output $y_{k+1}$ and construct the new data set $u_{k+1}, y_{k+1}, e_{k+1}$.

Step 3 Replace $k$ by $k+1$ and return to Step 1 .

Theorem 2 (Convergence Properties): Under these conditions defined above, the algorithm generates a sequence of tracking errors $\left\{e_{k}\right\}_{k \geq 0}$ that satisfies the monotonicity property (5) of NOILC. In more detail,

1) The error evolution is described by the relationship

$$
e_{k+1}=L_{k+1} e_{k}, \quad L_{k+1}=L\left(I-\beta_{k+1} G G^{*}\right),
$$

for all $k \geq 0$. Each $L_{k+1}$ is self adjoint and satisfies the inequality, for some $\mu_{k+1}>0$,

$$
-\left(1-\mu_{k+1}\right) L \leq L_{k+1} \leq L \leq I \text {. }
$$

2) If $\beta_{k+1}=\beta$ is iteration independent, then the error sequence converges in norm to the orthogonal projection of $e_{0}$ onto the kernel of $G G^{*}$. In addition,

a) If $\operatorname{ker}\left[G G^{*}\right]=\{0\}$, then

$$
\lim _{k \rightarrow \infty}\left\|e_{k}\right\| \mathcal{Y}=0
$$

b) If $e_{0}$ is in the range of $\left(I-L_{1}\right)$, then

$$
\sum_{k \geq 0}\left\|e_{k}\right\|_{\mathcal{Y}}^{2}<\infty .
$$

The range of $\left(I-L_{1}\right)$ is exactly the range of $G G^{*}$. 3) Similar convergence properties hold if, in addition, the gains $\beta_{k+1}$ satisfy a stronger condition of the form, for some scalar $\delta^{2}>0$,

$$
\delta^{2}<\beta_{k+1}<2\left(1-\delta^{2}\right)\left\|G^{*}\right\|^{2}, \quad k \geq 0 .
$$

Proof: The monotonicity property and the error evolution follow from the analysis preceding the theorem. $L_{k+1}$ is selfadjoint as it is a function of the self-adjoint operator $G G^{*}$. The existence of $\mu_{k+1}$ is a consequence of the properties $0<L=L^{*} \leq I$ and the gain range (8). If $\beta_{k+1}$ is iteration independent, the existence and properties of the limit error then follow directly from Theorem 5.9 in the text by Owens [26]. The general case when $\beta_{k+1}$ is iteration dependent is more complex and will be published separately. The simplest case is when $\beta_{k+1}$ varies but is iteration independent for iterations with index $k \geq k^{\prime}$. For example, if $e_{0}$ is in the 
range of $G G^{*}$, all the following $e_{k}$ lie in that subspace and, by considering the partial sequence $\left\{e_{k}\right\}_{k \geq k^{\prime}}$, it follows that the summability condition (20) must hold and hence that the error convergence (19) also holds. This ends the proof presented in this paper.

Note that the preceding discussion ensures that the new algorithm produces greater reductions in error norm each iteration than a pure NOILC algorithm. This observation is further supported by the operator ordering (18) and the consequent product ordering

$$
\Pi_{j=0}^{k} L_{j+1}<L^{k+1} \leq I, \quad k \geq 0 .
$$

The nature of this convergence and its dependence on gain parameters is discussed in the next subsection in terms of the spectrum of the $L_{k+1}$.

\section{B. Spectral Properties of the Convergence}

Just as the eigenvalues of a matrix $M$ govern the behaviour of an iteration $z_{k+1}=M z_{k}, k \geq 0$, the properties of the algorithm presented in this paper are related to spectral properties of the operators $\left\{L_{k+1}\right\}_{k \geq 0}$. Using the notation $\operatorname{spec}[\Gamma]$ to denote the set of spectral values of an operator $\Gamma: \mathcal{Y} \rightarrow \mathcal{Y}$, then the spectrum of $L_{k+1}$ is obtained from the Spectral Mapping Theorem to be exactly

$$
\operatorname{spec}\left[L_{k+1}\right]=\left\{\frac{1-\beta_{k+1} \eta}{1+\epsilon^{-2} \eta}: \eta \in \operatorname{spec}\left[G G^{*}\right]\right\}
$$

Consider the simplest case when all spectral values are eigenvalues and that $\left\{\eta_{j}\right\}_{j \geq 1}$ and $\left\{v_{j}\right\}_{j \geq 1}$ are the eigenvalues and orthonormal eigenvectors of $G G^{*}$. Then $L_{k+1}$ has eigenvectors $\left\{v_{j}\right\}_{j \geq 1}$ and

$$
L_{k+1} v_{j}=\frac{1-\beta_{k+1} \eta_{j}}{1+\epsilon^{-2} \eta_{j}} v_{j}, \quad j \geq 1 .
$$

The range of eigenvalues is given by

$$
0 \leq \eta_{j} \leq\left\|G^{*}\right\|^{2}
$$

and, if the eigenvalues are ordered $\eta_{1} \geq \eta_{2} \geq \eta_{3} \geq \cdots$, the largest eigenvalue $\eta_{1}=\left\|G^{*}\right\|^{2}$. In particular this means that (8) is just

$$
0 \leq \beta_{k+1} \leq 2 \eta_{1}^{-1}
$$

This characterization throws considerable light on the choice of gains $\beta_{k+1}$. Any gain in the range defined by (8) can be used and any gain in that range has an effect on the spectrum of $L_{k+1}$. In particular, in the absence of modelling error,

1) if $\beta_{k+1}=\eta_{j}^{-1}$, the contribution of the eigenvector $v_{j}$ to the error response is annihilated and remains zero for the remaining iterations. In addition, the contribution of all eigenvectors with eigenvalues close to $\eta_{j}$ are substantially reduced and continue to reduce as the iterations progress.

2) If, in addition, $\eta_{j} \geq \frac{1}{2} \eta_{1}$, the annihilation process is accompanied by an overall reduction in error norm.

This discussion shows that systematic and rapid reductions of the spectral content is possible whilst simultaneously reducing the error norm, the only constraint being that this is only true for that part of the spectrum defined by the inequality

$$
\frac{1}{2}\left\|G^{*}\right\|^{2} \leq \eta \leq\left\|G^{*}\right\|^{2}
$$

This is a spectral equivalent of a bandwidth condition as low eigenvalues $\eta_{j} \ll \eta_{1}$ are associated with, amongst other things, high frequency behaviours. This is quantified more precisely in Owens [26] for discrete state space systems. The details are omitted for brevity.

In practice, the eigenstructure of $G G^{*}$ will not be known. Despite this fact, the functional dependence of the spectrum of $L_{k+1}$ on that of $G G^{*}$ can be used to suggest parameter choices. For example, if the initial error $e_{0}$ has the form

$$
e_{0}=\sum_{j \geq 0} a_{j} v_{j}
$$

NOILC iterations produce errors that can be written in the form

$$
e_{k}=\sum_{j \geq 0} a_{j} v_{j}\left(\frac{1}{1+\epsilon^{-2} \eta_{j}}\right)^{k}
$$

which shows that the contribution of each and every eigenvalue/eigenvector reduces geometrically as iterations progress. In contrast,

1) the choice of $\beta_{k+1}=\left\|G^{*}\right\|^{-2}=\eta_{1}^{-1}$ for all iterations (the largest eigenvalue), then

$$
e_{k}=\sum_{j \geq 0} a_{j} v_{j}\left(\frac{1-\eta_{j} \eta_{1}^{-1}}{1+\epsilon^{-2} \eta_{j}}\right)^{k}
$$

indicating the immediate elimination of $v_{1}$, very rapid reductions of the contributions of the very largest eigenvalues (close to $\eta_{1}$ ) and improved reductions in small eigenvalues when compared with NOILC (obtained by setting $\beta_{k+1}=0$ ).

Note 2: For SISO discrete state space systems, (10) indicates that the choice of $\beta_{k+1}=\eta_{1}^{-1}$ will tend to eliminate frequencies close to that generating the maximum gain which will be the DC component or a resonance!

2) Suppose that the interval defined by (27) is divided up into $M \geq 1$ equally separated points $\left\{p_{j}\right\}_{1 \leq j \leq M}$ and that, with some ordering, the gains are chosen using $\beta_{k+1}=p_{k}^{-1}$, then

$$
e_{k+1}=\sum_{j \geq 0} a_{j} v_{j} \Pi_{q=1}^{k}\left(\frac{1-\eta_{j} p_{q}^{-1}}{1+\epsilon^{-2} \eta_{j}}\right)^{k}
$$

This shows that the eigenvectors corresponding to all eigenvalues in the range (27) are almost annihilated in the chosen set of $M$ ILC iterations.

Note 3: For SISO discrete state space systems, (10) indicates that this procedure will tend to eliminate frequencies in a (possibly wide) range around the DC or resonant frequency.

The potential of the acceleration mechanism is suggested by the above although more research is needed to improve the choice and assess the affect on other issues such as 
robustness. Some numerical examples are given in the next section.

\section{NUMERICAL EXAMPLES}

\section{A. Annihilating a Single Frequency/Spectral Value}

In [26], it is shown that, for a discrete SISO state space system $(A, B, C)$ operating on an interval $t=0,1,2, \cdots, N$ with transfer function $G(z)$ and a suitable choice of sinusoidal vector $\alpha(z)=\left\{1, z, z^{2}, \cdots, z^{N}\right\}$, the relation

$$
\left(G G^{*}-R^{-1} Q|G(z)|^{2} I\right) \alpha(z) \approx 0,
$$

with good accuracy if control in on a long enough interval. The interpretation of this is that $\alpha(z)$ is an approximate eigenvector of $G G^{*}$ with approximate eigenvalue $R^{-1} Q|G(z)|^{2}$. This suggests that the choice of

$$
\beta_{1}=R Q^{-1}|G(z)|^{-2}
$$

will almost annihilate an initial error $\alpha(z)$ in one iteration. To illustrate this idea, consider the linear SISO discrete system with transfer function

$$
G(z)=\frac{0.7}{z-0.7}, \quad \sup _{|z|=1}|G(z)|=2.333 .
$$

Zero initial conditions are assumed and a reference signal defined by the oscillating time series

$$
r=\{r(t)\}_{0 \leq t \leq N}, \quad r(t)=\sin \left(\frac{\pi}{2} t\right)=\operatorname{Im}\left[i^{t}\right],
$$

with a frequency $z=i$. The time interval chosen has length $N=200$.

Using discrete optimal ILC with weights $Q=R=1$ in the performance index avoids high gain feedback. Approximate annihilation of the single frequency reference is approached by using (33) to choose $\beta_{k+1}=3 \approx|G(i)|^{-2}$. The algorithm is initiated with $u_{0}=0$. The first iteration gives the error norm

$$
\left\|e_{0}\right\|=\|r\|=10,\left\|e_{1}\right\|=0.6328 .
$$

The substantial reduction in error provides a simple illustration of the approximate annihilation properties of the new algorithm. The gain used does not satisfy (8) and is not necessarily an acceptable choice for further iterations.

\section{B. Annihilating a Frequency/Spectral Band}

Reference signals, in most cases, contain a wide band of frequencies and plant dynamics are more complex. A simple illustration of possible approaches to such cases is the choice of

$$
G(z)=\frac{0.64}{(z-0.2)^{2}}
$$

with zero initial conditions and a reference signal (shown in Fig 1)

$$
r(t)=\sin ^{2}\left(\frac{\pi t}{20}\right)\left(1-\exp \left(-\frac{t}{10}\right)\right) .
$$

Again take $Q=R=1$ and $N=200$. Then (10) suggests that $\left\|G^{*}\right\|^{2} \approx 1$. Taking the initial control $u_{0}=0$, the results obtained from the use of NOILC are shown in the plot of

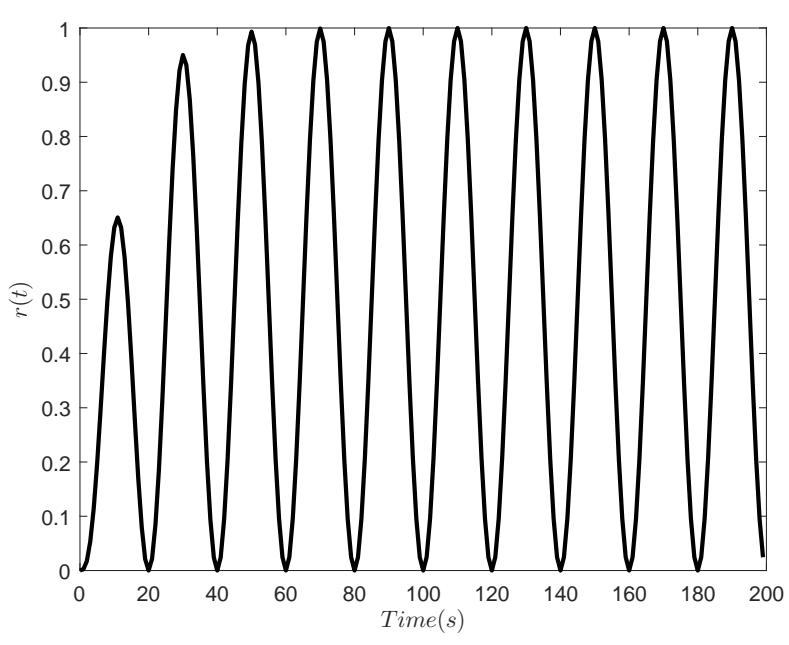

Fig. 1. The reference signal $r(t)$

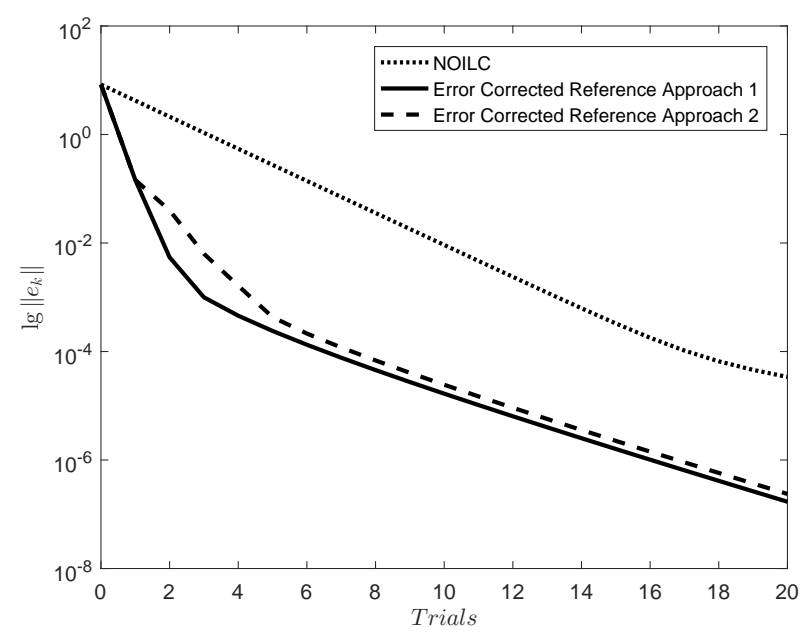

Fig. 2. Tracking error norm convergence for NOILC and the proposed algorithm

the evolution of the error norm in Fig 2. Acceleration of the algorithm is now approached using two approaches:

1) Approach One: Take $\beta_{k+1}=\beta$ to be iteration independent and let $\beta=1$ to approximately annihilate the contribution of the eigenvectors corresponding to the largest eigenvalues of $G G^{*}$. Alternatively, using (32), the choice will approximately annihilate the DC and low frequency bandwidth content of the error. The results for this error corrected algorithm are given in Fig 2 together with the NOILC result. The improvements in rate of convergence are clear and, given the simplicity of the error correction mechanism, well worth the use of the modification.

2) Approach Two: By letting the gains vary from iteration to iteration, different frequency contributions can be approximately annihilated. The constraint of (27) is taken to ensure that overall error reduction is guaranteed as the iterations progress. This means that we must 
focus on the frequencies where

$$
0.5 \leq|G(z)|^{2} \leq 1
$$

The values of these frequencies are not required for the choice of $\beta_{k+1}$. All that is needed is to choose $\beta_{k+1} \in$ $[1,2]$. To illustrate the possibilities, the choices are made to 'cover' this interval, for example,

$\beta_{1}=1.0, \beta_{2}=0.5, \beta_{3}=0.75, \beta_{4}=0.6, \beta_{5}=0.9$

and $\beta_{k+1}=1$ thereafter. The resulting algorithm behaviour is shown in Fig 2 including the basic NOILC behaviour. Again, the simple error corrected reference mechanism has accelerated the algorithm producing rapid reductions in error norm.

The examples provide evidence that the acceleration produced by the error correction mechanism, in the absence of modelling errors, can be substantial.

\section{CONCLusions}

The paper has shown that a combination of NOILC and Gradient ILC yields a modified NOILC algorithm that can achieve rapid convergence rates without the need for 'high gain' solutions (represented by the situation when $\epsilon^{2}$ becomes very small). The mechanism is simple and easy to implement and consists of a process of modifying the reference signal from iteration to iteration using the previously observed tracking error. The abstract nature of the approach chosen is that used by Owens [26] and shows that the ideas are quite general applying to a range of NOILC applications including continuous and discrete state space systems and the more general 'multi-task' problems introduced by Owens in [26].

In the simple form presented, a parameter $\beta_{k+1}>0$ is chosen on iteration $k+1$. It is generated from gradient considerations but choosing values can be, more usefully, related to the idea of annihilating the contribution of selected parts of the spectrum of $G G^{*}$ to the tracking error. For state space systems, this choice can be linked to the removal of frequency components from the error. Simple examples presented here verify the basic ideas and demonstrate the potential benefits of careful parameter selection. The examples are encouraging but future papers will address in more detail the design issues of parameter choice, the link to the form of plant dynamics and the observed retention of robustness (observed in simulations) using concepts similar to the robustness analysis of both Gradient ILC and NOILC provided in [26].

\section{ACKNOWLEDGMENT}

This work was partially supported by Science and Technology Innovation Team Project of Henan Province China (No 17IRTSTHN013), and ZZU-Southampton collaborative research project (Ref: 16306/01).

\section{REFERENCES}

[1] H.S. Ahn, K.L. Moore, and Y.Q. Chen. Iterative Learning Control: Robustness and Monotonic Convergence for Interval Systems. SpringerVerlag, London, 2007.

[2] D. A. Bristow, M. Tharayil, and A. G. Alleyne. A survey of iterative learning control: A learning-based method for high-performance tracking control. IEEE Control Systems Magazine, 26(3):96-114, 2006.

[3] K. L. Moore. Iterative Learning Control for Deterministic Systems. Advances in Industrial Control. Springer-Verlag, 1993.

[4] Z. Bien and $\mathrm{J} \mathrm{Xu}$. Iterative learning control: Analysis, integration, and application. Kluwer Academic Publishers, 1998.

[5] J.X. Xu, S.K. Panda, and T.H. Lee. Real-time iterative learning control: design and applications. Springer-Verlag, 2009.

[6] M. Norrlof. An adaptive iterative learning control algorithm with experiments on an industrial robot. IEEE Transactions on Robotics and Automation, 19(2):245-251, 2002.

[7] J. H. Lee and K. S. Lee. Iterative learning control applied to batch processes: An overview. Control Engineering Practice, 15:1306-1318, 2007.

[8] T. Liu and Y. Q. Wang. A synthetic approach for robust constrained iterative learning control of piecewise affine batch processes. Automatica, 48(11):2762-2775, 2012.

[9] C. T. Freeman. Control System Design for Electrical Stimulation in Upper Limb Rehabilitation. Springer International Publishing, 2016.

[10] S. S. Saab. Stochastic P-type/D-type iterative learning control algorithms. International Journal of Control, 76(2):139-148, 2003.

[11] C.J. Chien and A. Tayebi. Further results on adaptive iterative learning control of robot manipulators. Automatica, 44(3):830-837, 2008.

[12] R. W. Longman, Y.T. Peng, T. Kwon, H. Lus, R. Betti, and J.N. Juang. Adaptive inverse iterative learning control. Journal of the Chinese Society of Mechanical Engineers, 32(6):493-506, 2011.

[13] J. Ghosh and B. Paden. A pseudoinverse-based iterative learning control. IEEE Transactions on Automatic Control, 47(5):831-837, 2002.

[14] K. Kinoshita, T. Sogo, and N. Adachi. A relationship between iterative learning control using the gradient method and stable inversion. Transactions of the Society of Instrument and Control Engineers, 36(12):1117-1124, 2000

[15] D. H. Owens, J.J. Hatonen, and S. Daley. Robust monotone gradientbased discrete-time iterative learning control. International Journal of Robust and Nonlinear Control, 19(6):634-661, 2009.

[16] N. Amann, D. H. Owens, and E. Rogers. Iterative learning control using optimal feedback and feedforward actions. International Journal of Control, 65(2):277-293, 1996.

[17] S. Gunnarsson and M. Norrlof. On the design of ILC algorithms using optimization. Automatica, 37(12):2011-2016, 2001.

[18] D. De Roover and O. H. Bosgra. Synthesis of robust multivariable iterative learning controllers with application to a wafer stage motion system. International Journal of Control, 73(10):968-979, 2000.

[19] K.L. Moore, H.S. Ahn, and Y.Q. Chen. Iteration domain $H_{\infty}$-optimal iterative learning controller design. International Journal of Robust and Nonlinear Control, 18(10):1001-1017, 2008.

[20] B. Cichy, K. Galkowski, and E. Rogers. 2D systems based robust iterative learning control using noncausal finite-time interval data. Systems \& Control Letters, 64:36-42, 2014.

[21] Y.Wang and Z. Hou. Terminal iterative learning control based station stop control of a train. International Journal of Control, 84(7):12631277, 2011.

[22] J. Park, P. H. Chang, H. S. Park, and E. Lee. Design of learning input shaping technique for residual vibration suppression in an industrial robot. IEEE/ASME Transactions on Mechatronics, 11(1):55-65, 2006

[23] C. T. Freeman and Y. Tan. Iterative learning control with mixed constraints for point-to-point tracking. IEEE Transactions on Control Systems Technology, 21(3):604-616, 2013.

[24] P. Janssens, G. Pipeleers, and J. Swevers. A data-driven constrained norm-optimal iterative learning control framework for LTI systems. IEEE Transactions on Control Systems Technology, 21(2):546-551, 2013.

[25] T. D. Son, H. S. Ahn, and K. L. Moore. Iterative learning control in optimal tracking problems with specified data points. Automatica, 49(5):1465-1472, 2013.

[26] D.H. Owens. Iterative Learning Control: An Optimization Paradigm. Springer, 2015. 